\title{
CBF transcription factors involved in the cold response of Camellia japonica (Naidong)
}

\author{
Menglong Fan ${ }^{1}$, Rui Zhou ${ }^{1}$, Qinghua Liu ${ }^{1}$, Yingkun Sun ${ }^{\text {Corresp. } 1}$ \\ ${ }^{1}$ College of Landscape Architecture and Forestry, Qingdao Agricultural University, Qingdao, shandong, china \\ Corresponding Author: Yingkun Sun \\ Email address: sunyk678@qau.edu.cn
}

CBFs belong to the ERF subfamily of the AP2 supergene family and often play an important role in the cold acclimation of temperate plants. However, the role of CBFs in Camellia japonica (Naidong), the onlyCamelliajaponica population found in the temperate zones of China, remains unclear. It is very important to study the genetic composition of $C$.japonica (Naidong) to adapt to low temperature for Camellia species. Using full-length transcriptome data, we identified four CjCBF genes that respond to cold stress and analyzed their evolutionary relationships, domains, and expression patterns. The phylogeny of CBFs of 19 angiosperms divided the genes into three categories, and the four CjCBFs belong to a small subcluster. The strong response of CjCBF1 to cold treatment and its sustained high level of expression indicated that it plays an important role in the process of cold acclimation. A yeast two-hybrid assay revealed an interaction between CjCBF1, CjCBF2, and CjCBF5, and subcellular localization confirmed this finding. The expression of CjCBFs was tissue-specific: CBF1 was mainly expressed in leaves, and CBF3 was mainly expressed in stem. The responses of the four CjCBFs to drought and high temperature and the effect of light were also characterized. Our study provides new insight into the role of CBFs in the cold response in $C$. japonica (Naidong). 


\section{CBF transcription factors involved in the cold response of}

2

3 MengLong Fan ${ }^{1}$, Rui Zhou ${ }^{1}$, QingHua Liu' ${ }^{1}$, YingKun Sun ${ }^{1 *}$

4 1College of Landscape Architecture and Forestry, Qingdao Agricultural University

$5{ }^{*}$ Corresponding authors: YingKun Sun ${ }^{1 *}$

6 Street Address, College of Landscape Architecture and Forestry, Qingdao Agricultural University,

7 Qingdao 266109, Shandong, People's Republic of China

Email address:sunyk678@qau.edu.cn

9 Abstract

CBFs belong to the ERF subfamily of the AP2 supergene family and often play an important role in the cold acclimation of temperate plants. However, the role of CBFs in Camellia japonica (Naidong), the only Camellia japonica population found in the temperate zones of China, remains unclear. It is very important to study the genetic composition of C.japonica (Naidong) to adapt to low temperature for Camellia species. Using full-length transcriptome data, we identified four $\mathrm{CjCBF}$ genes that respond to cold stress and analyzed their evolutionary relationships, domains, and expression patterns. The phylogeny of CBFs of 19 angiosperms divided the genes into three categories, and the four CjCBFs belong to a small subcluster. The strong response of $C j C B F 1$ to cold treatment and its sustained high level of expression suggested that it plays an important role in the process of cold acclimation. A yeast two-hybrid assay revealed an interaction between $C j C B F 1, C j C B F 2$, and $C j C B F 5$, and subcellular localization confirmed this finding. The expression of $\mathrm{CjCBFs}$ was tissue-specific: $\mathrm{CBF} 1$ was mainly expressed in leaves, and CBF3 was mainly expressed in stem. The responses of the four CjCBFs to drought and high temperature and the effect of light were also characterized. Our study provides new insight into the role of CBFs in the cold response in C. japonica (Naidong).

\section{Introduction}

Low temperature stress is an important abiotic stress that greatly affects the distribution of plants and social and economic development worldwide. The study of cold resistance mechanisms is 
29

reduce the damage caused by low temperature through complex mechanisms, such as physiological metabolic pathways and molecular regulation (Agarwal et al, 2006a). Temperate plants can improve their cold tolerance by experiencing low, non-freezing temperatures (i.e., cold acclimation). This process involves stress perception, signal transduction, and the regulation of protein-coding genes, in which transcription factors act as important regulatory hubs that control a large number of genes to mediate the response to different stresses.

C-repeat binding factors (CBFs) belong to the ERF family of the AP2 supergene family, which has a common DNA-binding domain and two conserved motifs. They are distinguished by two signature sequences (PKK/RPAGRxKFxETRHP and DSAWR) (Haake et al, 2002). In some plants, CBFs have been shown to be key regulators in the cold acclimation pathway (Stockinger et al,1997;Medina et al,1999; Tayeh et al,2013;Francia et al,2016;Würschum et al,2017) and are specifically associated with the C-repeat/dehydration-responsive motif (CRT/DRE;

$\mathrm{G} / \mathrm{ACCGAC}$ ) of the promoter region of the COR gene(Lata\&Prasad,2011), which can mobilize a large number of genes involved in cold acclimation independent of the ABA pathway(Kodama et al,2000;Graham et al;1997;Ashraf et al,2007;Kornyeyev et al,2011). CBF transcription factors have been shown to improve cold resistance in maize, potato, tomato, Arabidopsis, tobacco, and other plants through overexpression verification(Ito et al,2006; Gilmour et al;2000; Liu et al,2011;Dubouzet et al,2003;Mei et al,2009;Al-Abed et al,2007;Tang et al,2011;Hsieh et al,2002;Hsieh et al,2002). In addition, previous studies have shown that CBF (DREB) not only participates in low temperature stress but also responds to drought and saline-alkali stress.

However, the overexpression of CBF transcription factors can inhibit plant growth and development and even lead to sterility (Tillett et al, 2012). This problem can be solved by incorporating different promoters into overexpression vectors with $C B F$ genes of different species. For example, the incorporation of Arabidopsis DREB1A and rd29A into an expression vector was not observed to significantly inhibit growth and sterility (Kasuga $M$ et ak, 1999). $A t C B F 1, A t C B F 2$, and AtCBF3 in A.thaliana are not only involved in the regulation of cold acclimation but also basic cold tolerance; however, $A t C B F 2$ appears to be more important than $A t C B F 1$ and $A t C B F 3$ in the process of cold acclimation (Zhao et al,2016). In Arabidopsis thaliana, $\mathrm{CBF} 2$ negatively regulates $\mathrm{CBF} 1$ and $\mathrm{CBF} 3$, but $\mathrm{CBF} 1$ and $\mathrm{CBF} 3$ do not directly regulate the expression of other $\mathrm{CBFs}$; instead, they coordinate the regulation of some downstream target genes (Novillo, Medina\&Salinas,2007). Thus, studying the combined expression of multiple genes is essential for improving plant cold resistance.

Camellia plants are mostly distributed in the subtropics and are not tolerant of low temperatures. Temperature greatly affects the economic value of Camellia plants. C. japonica (Naidong), which is the most northerly distributed Camellia species in China, has excellent cold resistance, but molecular research has been limited because of a lack of genomic sequences. Transcriptomic study of $C$. japonica (Naidong) has shown that $\alpha$-jasmonic acid may play an upstream signaling role in the response to cold stress( $\mathrm{Li}$ et al, 2016). However, no studies have identified $C B F$ genes in C. japonica (Naidong) nor have characterized their expression patterns in the response to cold stress. 
69 Here, we identified and isolated four low temperature-responsive CjCBFs transcription factors

70

from low temperature treatment full-length transcriptome data (PacBio). Structure analysis, phylogenetic analysis, Gene Ontology (GO) annotation, and the expression patterns of genes were clarified by bioinformatics technology, and the role of CjCBFs in the response to low temperature stress was characterized in detail. Overall, this study provides valuable information that could be used to improve the cold tolerance of Camellia plants.

\section{Materials and Methods}

Identification of new CjCBFs genes in C.japonica (Naidong) involved in cold

\section{tolerance}

We used our C.japonica (Naidong) low temperature and drought full-length transcriptome data based on PacBio and Illumina sequencing (PRJNA689105) as the local database. The protein sequences of CBF family genes (i.e., AtCBF1, AtCBF2, AtCBF3,AtCBF4) in Arabidopsis thaliana were queried to search the local databases using the BLASTP method with an E-value threshold of $<1 \mathrm{e}-20$. Then, HMMER3.0 (Potter, S. C. et al, 2018) was used to further screen sequences using default parameters $(\mathrm{E}<1 \mathrm{e}-5)$, the AP2 domain was based on Pfam database (Pfam accession, PF00847). The longest protein was selected as a representative for the gene. Sequences that do not contain the complete AP2 domain and signature sequences were eliminated.

\section{Sequence analysis}

ExPASy (Gasteiger, E et al, 2003) was used to analyze the relative molecular weight, isoelectric point, hydrophilicity, and other basic information of $\mathrm{CjCBFs}$ according to default parameters.

\section{Motif and phylogenetic analysis}

MEME (http://meme-suite.org/tools/meme) was used for the conservedmotif analysis of CjCBFs and AtCBFs proteins, with the maximum number of motifs set at ten. The parameters were as follows: minimum motif width, 6; maximum motif width, 50; Selected the motif classic mode and 0 -order model of sequences. MAFFT was used for sequence alignment according to default parameters (FASTA format, auto). The R package ggmsa was used to visualize the sequence alignment. In this study, C. japonica (Naidong) and 18 other plant species were selected from dicotyledons and monocotyledons, including Brachypodium distachyon, Oryza sativa and three additional monocotyledons, as well as Camellia japonica, Arabidopsis thaliana, Cucumis sativus and 19 other dicotyledons. The CBF-like protein data of the 19 species (with the exception of $C$. japonica) were downloaded from the NCBI Database. The protein sequences were selected by HMMER3.0. If the AP2 domain was truncated, or the AP2 domain match E-value exceeded 1e- 
1025 , the protein sequences were excluded. Based on this, $424 \mathrm{CBF}-$ like genes were identified from

103 the 19 plant species in order to construct the phylogeny tree. The phylogeny was constructed

104 using the maximum likelihood method with 1000 bootstrapping replicates in MEGA (v. 6.0)

105 (Tamura, K, 2013). The R package ggtree (v2.0.4) was used to visualize the phylogenetic tree.

106

107

108

109

110

111

112

113

114

115

116

117

118

119

120

121

122

123

124

125

126

127

128

129

130

131

132

133

\section{$\mathrm{GO}$ and interaction analysis}

KOBAS(Xie et al, 2010) (http://kobas.cbi.pku.edu.cn/) and A.thaliana (thale cress) were used for the GO enrichment analysis. The sequences of four $C j C B F \mathrm{~s}$ were inserted separately into pGADT7 Vector and pGBKT7 Vector; pGADT7-T+pGBKT7-Lam was the negative control and pGBKT7-53+pGADT7-T was the positive control. In order to verify self-activation, the pGADT7+ pGBKT7 - CjCBF1/CjCBF2/ CjCBF3/ CjCBF5 plasmid was transformed into Y2HGold yeast competent cells, with $30 \mathrm{mg} / \mathrm{ml} \alpha$-gal employed to detect the self-activation. Furthermore, in order to determine the suitable concentration of AbA, the inhibitory selfactivated AbA concentrations of $0.20 \mu \mathrm{l} / \mathrm{ml}, 0.30 \mu \mathrm{l} / \mathrm{ml}, 0.50 \mu \mathrm{l} / \mathrm{ml}, 0.70 \mu \mathrm{l} / \mathrm{ml}$ and $0.90 \mu \mathrm{l} / \mathrm{ml}$ were adopted for the five working solutions. The pGBKT7 and pGADT7 + CjCBF1, pGBKT7 and pGADT7 + CjCBF2, pGBKT7 and pGADT7 + CjCBF3, and pGBKT7 and pGADT7 + CjCBF5 plasmids were transformed into Y2HGold yeast competent cells, respectively, to exclude false positives. Moreover, the pGADT7 $+C j C B F s$ and pGBKT7 $+C j C B F s$ plasmid was transformed into Y2HGold yeast competent cells to verify the interaction between $\mathrm{CjCBFs}$.

RNA-seq data

In order to explore the transcriptional expression of $\mathrm{CjCBFs}$ at low temperature stress across time, we evaluated our low temperature stress transcriptome (PRJNA689105) under a $4^{\circ} \mathrm{C}$ treatment lasting $0 \mathrm{~h}, 12 \mathrm{~h}, 24 \mathrm{~h}$, and $72 \mathrm{~h}$, with 4 groups of transcriptome data. The expression of the CjCBFs gene was analyzed and the results were visualized by the R package pheatmap. qRT-PCR technology was employed to verify the transcriptome data and the co-expression method was used to predict the potential lncRNA regulating CjCBFs ( $\mathrm{Li} \mathrm{J}$ et al, 2015).

\section{Subcellular localization}

Four DNA sequences encoding CBF proteins were inserted into the super1300-GFP overexpression vector, and Agrobacterium tumefaciens GV3101 containing super1300-CjCBF1, super1300-CjCBF2, super1300-CjCBF3, and super1300-CjCBF5 was injected into the Abaxial of tobacco (Nicotiana benthamiana) leaves (Li, Z.Q, 2013). A Leica DM2500-DM2500 LED fluorescence microscope was used to obtain fluorescence information.

Peer] reviewing PDF | (2021:04:59897:2:1:NEW 15 Aug 2021) 
134 Promoter cloning and Cis-Element analysis

135 C. japonica (Naidong) DNA was extracted via the CTAB method (Doyle JJ and Doyle JL, 136 1987) to clone the promoter. The $C j C B F 1$ upstream 5'UTR sequence was obtained 137 from the Takara Genome Walking Kit (Takara, Japan) following the manufacturer's 138 instructions. Design of nested PCR specific primers with primer 5.0 (Supplementary 139 Table S7). The obtained sequence was connected to a $\mathrm{T}$ vector for sanger sequencing 140 and the Cis-Elements in the sequence were analyzed based on the PlantCARE database 141 (Lescot M et al, 2002).

Plant materials and low temperature treatment

C. japonica (Naidong) seeds were obtained from the Qingdao Botanical Garden $\left(36^{\circ} 05^{\prime} \mathrm{N}\right.$, $120^{\circ} 08^{\prime}$ E), Qingdao Agricultural University Cooperative, China, in September 2018. The seeds were sown in sand in the Qingdao Agricultural University culture room $\left(20 \pm 2{ }^{\circ} \mathrm{C}\right)$ during the winter until the fourth true leaf appeared. Following germination, the plants were transferred into the artificial climate room of the university $\left(36^{\circ} 31^{\prime} \mathrm{N}, 120^{\circ} 39^{\prime} \mathrm{E}\right)$ under normal culturing conditions (plastic pots: top/bottom diameter $20 / 12 \mathrm{~cm}$, height $26 \mathrm{~cm}$; temperature: $25 \pm 2^{\circ} \mathrm{C}$; relative humidity: $60 \%$; soil: peat soil $80 \%$ + river sand $20 \%$; natural light) for 3 months.

For the tissue specific expression experiment, we selected seedlings of a similar growth status and divided them into two groups, with three plants per group. Each group was treated at $4^{\circ} \mathrm{C}$ for either $0 \mathrm{~h}$ or $24 \mathrm{~h}$ in a low temperature incubator. Tender roots, stems, leaves, buds, and flowers were collected at $0 \mathrm{~h}$ and $24 \mathrm{~h}$. For the heat, cold and light treatments, seedlings were separated into several groups. The first group was placed at $40^{\circ} \mathrm{C}$ (from $25^{\circ} \mathrm{C}$ to $40^{\circ} \mathrm{C}$ with a $1^{\circ} \mathrm{C}$ increment per hour) and maintained for $24 \mathrm{~h}$ (2000lx light intensity and $60 \%$ humidity). The second group was placed at $40^{\circ} \mathrm{C}$ (from $25^{\circ} \mathrm{C}$ to $40^{\circ} \mathrm{C}$ with a $1{ }^{\circ} \mathrm{C}$ increment per hour) and maintained for $24 \mathrm{~h}(60 \%$ humidity). The plants were wrapped with foil to avoid light. The third group was placed at $4^{\circ} \mathrm{C}$ (from $25^{\circ} \mathrm{C}$ to $40^{\circ} \mathrm{C}$ with a $1^{\circ} \mathrm{C}$ increment per hour) and maintained for $24 \mathrm{~h}(20001 \mathrm{x}$ light intensity). The fourth group was placed at $4^{\circ} \mathrm{C}$ (from $25^{\circ} \mathrm{C}$ to $40^{\circ} \mathrm{C}$ with a $1^{\circ} \mathrm{C}$ increment per hour) and maintained for $24 \mathrm{~h}$. ( 1 degree per hour from $25^{\circ} \mathrm{C}$ to $\left.4^{\circ} \mathrm{C}\right)$. The plants were wrapped with foil to avoid light. The drought treatment involved stopping watering for 20 days and measuring the relative soil water content to determine the drought degree (Supplementary Table S8). Unstressed seedlings were used as the control samples (CK). Three biological replicates were collected from the same position at each collection time for RNA isolation.

\section{RNA extraction and Expression analysis of qRT-PCR}

The total RNA extraction was performed using the SPARKeasy A0305 kit (QingDao, China), following the manufacturer's instructions. Each treatment comprised three replications. The 
169 integrity and concentration were detected by 1\% agarose gel electrophoresis and a

170 NanoDrop2000 (Thermo Fisher Scientific, Waltham, MA, USA). First-strand cDNA was

171 prepared using the Takara PrimerScriptTMRT reagent kit with gDNA Eraser, and RT-qPCR

172 primers were designed by Primer 5.0 (Supplementary Table S7). Takara TB Green was used to

173 set up the Premix Ex Taq qGreTli RNaseH Plus for the qRT-PCR experiment. Per the

174 manufacturer's instructions, $18 S$ was used as an internal reference gene, and there were three

175 biological replicates and three technical replicates in each group. The relative expression was

176 determined by a three-step method $\left(95^{\circ} \mathrm{C}\right.$ for $5 \mathrm{~s}, 55^{\circ} \mathrm{C}$ for $[15 \mathrm{~s}]$, and $72^{\circ} \mathrm{C}$ for $30 \mathrm{~s}$, repeated 40

177 times) on an Applied Biosystems StepOnePlus. The gene expression level was calculated

178 according to the quantitative $\mathrm{Ct}$ method. The R package pwr was used to conduct Student's $t$ -

179 tests. The R packages corrplot (0.84) and circlize (version 0.4.11) were used to conduct

180 correlation analysis.

181

182

183

184

185

186

187

188

189

190

191

192

193

194

195

196

197

198

199

200

201

202

203

204

\section{Results}

\section{Identification and characterization of $\mathrm{CjCBFs}$}

First, we used the transcriptome data of $C$. japonica (Naidong) as the local blast resource bank to obtain 11 candidate genes. Further screening incorrectly predicted CBF genes and redundant sequences, and four CBF transcription factors responsive to low temperature were identified. They were named according to their homology with CBF transcription factors in related plants (Camellia sinensis). The nucleotide and amino acid sequences are provided in Supplementary Table S1. The full lengths of the $C j C B F$ s sequences are $1.82 \mathrm{~kb}, 1.25 \mathrm{~kb}, 1.42 \mathrm{~kb}$, and $1.35 \mathrm{~kb}$. The CDSs are between 540 and $837 \mathrm{bp}$, and the number of amino acids are between 179 and 278 . The molecular weights of the four cjCBF proteins are $30282.9 \mathrm{kDa}, 19449.25 \mathrm{kDa}, 26655.03$ $\mathrm{kDa}$, and $26526.23 \mathrm{kDa}$. The isoelectric point ranges from 4.9 to 9.8 . Hydrophilicity ranges from 3.02 to 1.8 (Table. 1).

\section{Analysis of conserved motifs}

A comparison of the CjCBFs sequences (Supplementary Fig. 1) revealed that the internal similarity of CjCBFs was $73.96 \%$, and the domain sequence was highly conserved. Comparison of the domain sequences of A. thaliana and C. japonica (Naidong) (Fig. 1A) showed that the domain of $C$. japonica (Naidong) consists of the AP2 family universal DNA-binding domain (AP2 domain) and two signature sequences (DS-W-L), which were slightly different from the conserved domain of $A$. thaliana. We then analyzed the conserved motifs of $A$. thaliana and $C$. japonica (Naidong) and identified 10 motifs (Fig. 1B) with amino acid lengths between 6 and 50 . In particular, Motif10 (WYQGDD) was the shortest (6 residues), while Motif1 (KKVRETRHPIYRGVRQRNSGKWVCEVREPNKKSRIWLGTFPTAEM AARAH) and motif2 (DVAAJALRGRSACLNFADSAWRLPIPESLCPKDIQKAA AEAAEAFRPELC) were the longest (50 residues) and contained the AP2 domain. Only CjCBFs have motif8 
205 (YLDVSGDVAKP), and only $A$. thaliana has motif7 (TTDHGLDMEETLVE). Only CjCBF1

206 and CjCBF5 have motif10. The functions of most of the motifs have not yet been elucidated. The 207 similarity of motifs within species indicates that CBFs within species are highly conserved. The 208 composition of different conserved motifs may be related to variation in the functional 209 differentiation of genes.

210 Analysis of the phylogenetic tree

211 To characterize the evolutionary relationships of $C B F \mathrm{~s}$ among species, we constructed a genetic 212 tree (Fig. 2) using 424 CBFs from 19 species, including dicotyledons and monocotyledons.

213 These genes were generally divided into three categories. Monocotyledons and dicotyledons

214 were clearly separated, and dicotyledons were divided into two subclasses: Clade I and Clade

II . However, some monocotyledons (e.g., oil palm) were divided into A- II subcluster in Clade

I, and rice was also partially distributed in this subcluster. This cluster is close to Clade III, with groups containing only Monocotyledons. This may represent the region of functional overlap of dicotyledon and monocotyledon CBFs. This also indicates that this cluster of $C B F$ ancestral genes had differentiated before the separation of monocotyledons and dicotyledons and thus that the function and pedigree of $C B F s$ became gradually enriched. A- I and B-II were the largest subclusters in Clade I and Clade II, respectively. CjCBFs were divided into A- I subclusters and clustered with $A$. thaliana. $C B F s$ were highly conserved within species, and $C B F s$ of the same species often clustered together.

GO enrichment analysis of $C j C B F s$ was conducted to better understand the biological function of CjCBFs. CjCBFs were enriched in nucleus (GO:0005634), DNA-binding transcription factor activity (GO:0003700), regulation of transcription, DNA-templated (GO:0006355), transcription regulatory region sequence-specific DNA binding (GO:0000976), glucosinolate metabolic process (GO:0019760), cold acclimation (GO:0009631), and response to cold (GO:0009409). The results of the $\mathrm{GO}$ analysis confirmed that $\mathrm{CjCBFs}$ play a role in cold acclimation $\left(4^{\circ} \mathrm{C}\right)(\mathrm{Fig}$. 3) (Xie et al, 2010).

\section{Expression model of $C j C B F s$ based on RNA-seq}

Based on the data analysis of the full-length transcriptome under treatment with low temperature stress, the standardized FPKM cluster analysis heat map (Fig. 4A) shows that CjCBFs responded strongly to low temperature. Although $C j C B F 1$ played a role in all three gradient treatment 
236

237

238

239

240

241

242

243

244

245

246

247

248

249

250

251

252

253

254

255

256

257

258

259

260

261

262

263

264

265

266

267

268

269

270

271

272

groups, it was significantly up-regulated at the $12 \mathrm{~h}$ stage, and the strength of the effect was significantly higher in the $12 \mathrm{~h}$ stage than in the $24 \mathrm{~h}$ and $72 \mathrm{~h}$ stages. The response of $C j C B F 3$ to low temperature was basically the same in the three treatment groups. $C j C B F 2$ responded in all three treatment groups but was significantly up-regulated in $24 \mathrm{~h}$ and then decreased in $72 \mathrm{~h}$. The expression of $C j C B F 3$ and $C j C B F 5$ was similar to that of $C j C B F 1$, except that $C j C B F 5$ was significantly up-regulated in $24 \mathrm{~h}$ but then decreased significantly and remained expressed at a low level. qRT-PCR was used to verify the relative expression of $C j C B F s$. The pattern was nearly identical, indicating that our transcriptome results were reliable (Fig.5C). The correlation analysis also supports this finding (Fig. 4B). There is much evidence showing that lncRNA participates in biological processes by regulating gene expression, including responses to stress in plants. Our SMART and next-generation sequencing data were used to predict possible lncRNA transcripts. A total of 147 possible $C j C B F$-containing transcripts (Fig. 4D) were predicted by lncRNA.

\section{Interactions of $\mathrm{CjCBFs}$}

To further study the post-transcriptional regulation mechanism of CjCBFs under cold stress, we characterized the interactions among CjCBF1, CjCBF2, CjCBF3, and CjCBF5 using the Y2Hgold yeast two-hybrid technique. All CjCBFs were completely inhibited when the concentration of Aureobasidin A (AbA) reached $90 \mathrm{ng} / \mu \mathrm{l}$, while the corresponding $\mathrm{AbA}$ concentration for $\mathrm{CjCBF} 2, \mathrm{CjCBF} 3$, and $\mathrm{CjCBF} 5$ was $30 \mathrm{ng} / \mu \mathrm{l}$ (Fig. 5A), The results of selfactivation verification showed that the self-activation ability of $\mathrm{CjCBF} 1$ was significantly higher than that of other CjCBFs, CjCBF2-PGAD, CjCBF3-PGAD, and CjCBF5-PGAD were transformed into yeast Y2HGold cells in CjCBF1-PGBD; PGAD-T and PGBD-lam were transformed into the negative control, and PGBD-T and PGBD-53 were transformed into the positive control. The results of interaction verification showed that the yeast cells co-transformed with $\mathrm{CjCBF} 1$ and $\mathrm{CjCBF} 2, \mathrm{CjCBF} 1$ and $\mathrm{CjCBF} 5$ could grow normally on the nutrient-deficient medium, indicating that $\mathrm{CjCBF} 1$ could interact with $\mathrm{CjCBF} 2$ and $\mathrm{CjCBF} 5$ (Fig. 5B). To verify the yeast two-hybrid results, we analyzed the subcellular localization of $\mathrm{CjCBF} 1, \mathrm{CjCBF} 2$, and CjCBF5. CjCBF1 and CjCBF5 were located in the nucleus and cell membrane, and CjCBF2 was located in the cell membrane (Fig. 5C). These results were consistent with the yeast two-hybrid results. Several CjCBFs were expressed to aid the ability of Camellia japonica to cope with low temperature stress.

\section{Expression of CjCBFs in five Camellia japonica tissues}

To more fully characterize the spatial expression characteristics of $C j C B F S$ in $C$. japonica (Naidong), the leaves, stems, flowers, buds, and roots of $C$. japonica (Naidong) were treated with $4^{\circ} \mathrm{C}$ for $24 \mathrm{~h}$. The expression of $C j C B F s$ was detected by qRT-PCR. the results showed, In the normal cultured $\mathrm{CK}$ group, the expression of $4 \mathrm{CjCBFs}$ was lower, but it was worth noting that the expression of $C j C B F 1, C j C B F 3$ in flowers was higher than that of $C j C B F 2, C j C B F 5$. This suggests 
that $C j C B F 1, C j C B F 3$ may be involved in flower development. after $24 \mathrm{~h}$ of $4^{\circ} \mathrm{C}$ treatment, 4 CjCBFs were Significant difference, but only $C j C B F 1$ was highly expressed in all five tissues, especially in the petals, and $C j C B F 2, C j C B F 3$, and $C j C B F 5$ were expressed at low levels. The expression of $C j C B F 2$ in roots and flowers was low, and the expression of $C j C B F 3$ and $C j C B F 5$ in stems, leaves, and buds was significantly higher, and the expression of $C j C B F 5$ in buds was higher (Fig. 6). These results suggest that $C j C B F 1$ may play an important role in the response to cold stress in C. japonica (Naidong).

\section{Bioinformatics Analysis of $C j C B F 1$ Cis-Elements}

Promoter cis-elements function as transcription factor binding sites and are key for the transcriptional regulation in response to abiotic stress (Moore M. J et al, 2010). As CjCBF1 plays an important role in $\mathrm{CjCBFs}$, we cloned and analyzed the $1400 \mathrm{bp}$ upstream sequences of CjCBF1. Bioinformatics analysis revealed the sequence to contain 31 cis-acting elements (Fig. 7 and Supplementary Table S3), including 12 photosensitive responses and related cis-elements, CGTCA and TGACG motifs (involved in methyl jasmonate responsiveness). The sequence was also rich in TC-rich repeats related to stress, and MYB and MYC related cis elements. The results suggest that $C j C B F 1$ has a wide response to abiotic stress and that light may play an important role in the expression of CjCBFs.

\section{Expression of CjCBFs under different stresses and the effect of light}

Camellia japonica (Naidong) was treated with high temperature stress, drought stress, low temperature stress, and light stress. Only $C j C B F 1$ and $C j C B F 5$ responded to high temperature $\left(40^{\circ} \mathrm{C}\right)$ stress(Fig. $\left.8 \mathrm{~A}\right)$, but the expression of $C j C B F 1$ was significantly lower under $40^{\circ} \mathrm{C}$ treatment than under $4^{\circ} \mathrm{C}$ for $24 \mathrm{~h}$ and decreased slightly under joint high temperature and light stress treatment. Under low temperature and shading stress(Fig. 8B), the expression of $C j C B F s$ decreased significantly compared with the non-shading treatment, which indicated that light may not represent a signal that $C j C B F s$ respond to at high temperature; however, the effect of light on $C j C B F s$ was more important at low temperature. The responses of $C j C B F 1, C j C B F 3$, and $C j C B F 5$ to drought stress were weak; only $C j C B F 2$ showed a significant response to drought stress, indicating that the responses of $C j C B F s$ in Camellia japonica to drought stress are not strong(Fig. 8C).

\section{Discussion}

When plants are subjected to abiotic stress, complex transcriptional and metabolic mechanisms are mobilized. Previous studies have shown that CBF transcription factors are essential for regulating responses to various stresses, and they can mobilize $35 \%$ of $C O R$ genes involved in cold acclimation independent of the ABA pathway. The ICE-CBF-COR pathway is widely known to be one of the core pathways in the response to low temperature stress in many plants, 
308 including A.thaliana. ICE1 and its homologous gene ICE2 dynamically regulate the expression 309 of $C B F s$ to ensure that they are expressed at appropriate levels in different periods. However, the 310 regulation of $C B F s$ is complex; for example, the promoter has at least one CRT/DRE motif COR gene, PHYTOCHROME-INTERACTING FACTOR4 (PIF4) and PIF7 (Barrero-Gil \& Salinas, 2018) and the brassinosteroid signaling transcription factors BRASSIN AZOLE RESISTANT1 (BZR1) (Eremina,Rozhon\& Poppenberger, 2016). In addition, the number of CBFs is often related to the size of the genome (Wang et al, 2019). Five CBF transcription factors have been identified in Camellia sinensis genomes, but the response patterns and characteristics of CBFs in C. japonica (Naidong) remain unclear. As the northernmost Camellia in China, exploration of the cold resistance mechanism of $C$. japonica (Naidong) is particularly important for enhancing the cold tolerance of Camellia. However, its genome was not sequenced. Using data from the PacBio and the Illumina full-length transcriptome, four $C j C B F s$ were identified. These $C j C B F s$ participated in the response to cold stress at non-freezing temperatures, and their expression was

\section{and drought stress.}

Plants have evolved several strategies to cope with environmental stress. The evolution of multiple copies of genes can permit plants to quickly respond to stress. Multiple copies of $C B F$ genes have often been observed(Zhao et al, 2016). However, there are no reference genome sequences of $C$. japonica (Naidong), precluding the possibility of determining the actual origin of these gene copies. In our transcriptome data, we detected multiple CBF transcripts, which have almost the same CDS region and significantly different UTR regions compared with ontological annotations. However, the expression levels of these copies were not the same. We speculate that the UTR region may also play an important role in the CBF pathway of coldresistant Camellia japonica. Comparison with the database revealed that lncRNAs are not predicted in the UTR region of $\mathrm{CjCBF}$; however, this does not mean that the UTR region is not involved in the response to low temperature stress. In the phylogenetic tree, $C j C B F 1$ and $C j C B F 3$ are clustered together, and $C j C B F 2$ and $C j C B F 5$ are clustered together. This, combined with the results of our yeast experiment, indicates that $C j C B F 1, C j C B F 2$, and $C j C B F 5$ show different patterns of expression and are regulated differently. $C j C B F 3$ appears to be a functional redundancy of $C j C B F 1$ in the response to low temperature stress and does not directly participate in the internal interaction. These results confirm the core role of $C j C B F 1$ in the response to low temperature stress. Several studies have shown that $C B F s$ are not only involved in the stress response but are also involved in the inhibition of growth and development and delayed flowering. Analysis of the expression in five Camellia tissues in the absence of cold treatment revealed that $C j C B F 1$ was highly expressed in flowers, but there was no significant change after cold treatment. Although $C B F$ plays an important role in stress, $C B F$ overexpression inhibits plant growth (Li et al, 2016; Zhao et al, 2016; Gilmour et al, 2004); there is thus a need to find ways to avoid the negative effects of overexpression of these genes.

We obtained the upstream 1400-bp sequence of $C j C B F 1$ by the walking method (Supplementary table.S2) and identified 12 homeopathic elements related to light, 1 cis-acting element involved 
348

349

350

351

352

353

354

355

356

357

358

359

360

361

362

363

364

365

366

367

368

369

370

371

372

373

374

375

376

377

378

379

380

381

382

in defense and stress responsiveness, and 8 cis-acting regulatory elements involved in MeJA responsiveness (Supplementary table.S3). There are several light-response homeopathic elements in $\mathrm{CjCBFs}$; We analyzed the response of $C j C B F$ s to light under stress in detail through a light control experiment under low temperature. We found that the expression of $C j C B F s$ decreased significantly under shading, indicating that light promotes the low temperature response of Camellia japonica.

However, light is not a necessary condition. Thus, the study of broad-spectrum and sensitive stress genes is particularly important and is the reason for increased interest in studying the effects of multiple types of stress. This suggests that the expression of $C j C B F s$ is indeed different between day and night in winter. Interestingly (Gilmour,Fowler\&Thomashow,2004), our light control experiment under high temperature stress revealed that light did not cause changes in the expression of $C j C B F s$, and light intervention did not affect the response of $C j C B F 1$ and $C j C B F 5$ to heat stress. We found that low temperature induces the strongest and most complex regulatory response of $C j C B F s$. In addition, there are MeJA signal elements in the promoter sequence of $C j C B F 1$, indicating that it may be regulated by jasmonic acid and may be involved in the metabolism of glucosinolates. $C B F$ overexpression in A.thaliana promotes the accumulation of glucose, sucrose, and proline, which aids the survival of plants at low temperatures.

$C B F s$ are structurally expressed in $A$. thaliana. $C B F 1$ and $C B F 3$ are the main components underlying the response to cold stress in plants(Gilmour et al,2000;Harmer et al,2000). $C B F 2$ is involved in the regulation of $C B F 3$ and $C B F 1$. We verified the relationship between $C j C B F s$. $C j C B F 1$ plays a central regulatory role. $C j C B F 1$ may be expressed first and then regulate $C j C B F 5$ and $C j C B F 2$. The expression patterns indicated that the three $C j C B F s$ were maintained in a dynamic balance, and the rapid up-regulation of $C j C B F 1$ caused by low temperature breaks this balance. $C j C B F 2$ and $C j C B F 5$ negatively regulate $C j C B F 1$ to maintain its level of expression within a specific range.

\section{Conclusion}

Four CjCBFs involved in the cold response of C. japonica (Naidong) were studied. An analysis of the motifs and evolution of these proteins provided information that could be useful for studying other Camellia species. The interaction mode of $C j C B F 1, C j C B F 2$, and $C j C B F 5$ provided new insight into the biological function of $\mathrm{CBF}$ transcription factors in Camellia. The exploration of various stresses and responses to light expanded the known functions of $\mathrm{CjCBF}$ transcription factors and will aid future studies of the mechanism of stress adaptation in Camellia and other plants. 
383 Supplementary Figure1. Sequence similarity analysis of CjCBFs

384 Supplementary Table S1. Sequencing of CjCBFs.

385 Supplementary Table S2. Sequencing of CjCBF1 promoter.

386 Supplementary Table S3. Analysis of the homeopathic elements of promoters.

387 Supplementary Table S4. Fig. 4c qRT-PCR.Raw data.

388 Supplementary Table S5. Fig. 6 qRT-PCR.Raw data.

389 Supplementary Table S6. Fig. 7 qRT-PCR.Raw data.

390 Supplementary Table S7. The primer of qRT-PCR and promoter clon.

391 Supplementary Table S8. The relative water content of soil.

392 References

Agarwal PK, Agarwal P, Reddy MK, Sopory SK. 2006a. Role of DREB transcription factors in abiotic and biotic stress tolerance in plants. Plant Cell Rep, 25(12):1263-74. DOI: 10.1007/s00299006-0204-8.

Al-Abed D, Madasamy P, Talla R, Goldman S, Rudrabhatla S. 2007. Genetic engineering of maize with the DREB1A/CBF3 gene using split-seed explants. Crop Sci, 47(6): 2390-2402. DOI:10.2135/cropsci2006.11.0712.

Ashraf M, Foolad MR. 2007. Roles of glycine betaine and proline in improving plant abiotic stress resistance. Environ Exp Bot, 59(2): 206-16. DOI:10.1016/j.envexpbot.2005.12.006. Barrero-Gil J, Salinas J. 2018. Gene regulatory networks mediating cold acclimation: The CBF pathway. Adv. Exp.Med. Biol, 1081: 3-22. DOI:10.1007/978-981-13-1244-1_1. 
404

405

406

407

408

409

410

411

412

413

414

415

416

417

418

419

420

421

422

423

424

425

426

427

428

429

430

tissues. Phytochem Bull. 19:11-15. DOI:10.1016/j.bse.2009.07.003.

Dubouzet J, Sakuma Y, Ito Y, Kasuga M, Dubouzet E, Miura S, Seki M, Shinozaki K, YamaguchiShinozaki K. 2003.OsDREB genes in rice, Oryza sativa L., encode transcription activators that function in drought-, high-salt-, and cold-responsive gene expression. Plant J, 33(4): 751-63. DOI:10.1046/j.1365-313x.2003.01661.x.

Eremina M, Rozhon W, Poppenberger B. 2016. Hormonal control of cold stress responses in plants. Cell Mol Life Sci, 73:797-810. DOI:10.1007/s00018-015-2089-6.

Francia E, Morcia C, Pasquariello M, Mazzamurro V, Milc JA, Rizza F, Terzi V, Pecchioni N. 2016. Copy number variation at the $\mathrm{HvCBF} 4-\mathrm{HvCBF} 2$ genomic segment is a major component of frost resistance in barley. Plant. Mol. Biol, 92(1-2):161-175. DOI:10.1007/s11103-016-05054.

Gasteiger E, Gattiker A, Hoogland C, Ivanyi I, Appel RD, Bairoch A. 2003. ExPASy: The proteomics server for in-depth protein knowledge and analysis. Nucleic Acids. 31: 3784-3788. DOI: $10.1093 /$ nar/gkg563.

Gilmour SJ, Sebolt AM, Salazar MP,Everard JD, Thomashow MF. 2000. Overexpression of the Arabidopsis CBF3 transcriptional activator mimics multiple biochemical changes associated with cold acclimation. Plant Physiol, 124(4):1854-65. DOI:10.1104/pp.124.4.1854.

Graham LA, Liou YC, Walker VK, Davies PL. 1997. Hyperactive antifreeze protein from beetles. Nature, 388(6644):727-8. DOI:10.1038/41908.

Gilmour SJ, Fowler SG, Thomashow MF. 2004. Arabidopsis Transcriptional Activators CBF1, CBF2, and CBF3 have Matching Functional Activities. Plant Mol Biol, 54(5): 767-781. DOI:10.1023/B:PLAN.0000040902.06881.d4.

Gilmour SJ, Sebolt AM, Salazar MP, Everard JD, Thomashowm MF. 2000. Overexpression of the Arabidopsis CBF3 transcriptional activator mimics multiple biochemical changes associated with cold acclimation. Plant Physiol, 124(4): 1854-1865. DOI:10.1104/pp.124.4.1854.

Harmer SL, Hogenesch JB, Straume M, Chang HS, Han B, Zhu T, Wang X, Kreps JA, Kay SA. 2000. Orchestrated transcription of key pathways in Arabidopsis by the circadian 
431

432

433

434

435

436

437

438

439

440

441

442

443

444

445

446

447

448

449

450

451

452

453

454

455

456

457

clock. Science, 290(5499): 2110-2113. DOI:10.1126/science.290.5499.2110.

Haake V, Cook D, Riechmann JL, Pineda O, Thomashow MF, Zhang JZ. 2002.Transcription Factor CBF4 Is a Regulator of Drought Adaptation in Arabidopsis. Plant Physiol, 130(2): 639648. DOI:10.1104/pp.006478.

Hsieh T, Lee J, Yang P, Chiu L, Charng Y, Wang Y, Chan M. 2002. Heterology expression of the Arabidopsis C-repeat/dehydration response element binding factor 1 gene confers elevated tolerance to chilling and oxidative stresses in transgenic tomato. Plant Physiol, 129(3): 1086-94. DOI:10.1104/pp.003442.

Hsieh T, Lee J, Charng Y, Chan M. 2002. Tomato plants ectopically expressing Arabidopsis CBF1 show enhanced resistance to water deficit stress. Plant Physiol, 130(2): 618-26. DOI:10.1104/pp.006783.

Ito Y, Katsura K, Maruyama K, Taji T, Yamaguchi-Shinozaki K. 2006. Functional analysis of rice DREB1/CBF-type transcription factors involved in cold-responsive gene expression in transgenic rice. Plant Cell Physiol, 47(1): 141-53. DOI:10.1093/pcp/pci230.

Kasuga M, Liu Q, Miura S, Yamaguchi-Shinozaki K, Shinozaki K. 1999. Improving plant drought, salt and freezing tolerance by gene transfer of a single stress-inducible transcription factor. Nat. Biotechnol, 17(3): 287-291. DOI: 10.1093/pcp/pch037.

Kodama H, Nishiuchi T, Seo S, Ohashi Y, Iba K. 2000. Possible involvement of protein phosphorylation in the wound-responsive expression of Arabidopsis plastid $\omega-3$ fatty acid desaturase gene. Plant Sci, 155(2): 153-60. DOI:10.1016/s0168-9452(00)00210-7.

Kornyeyev D, Logan BA, Payton P, Allen RD, Holaday AS. 2001. Enhanced photochemical light utilization and decreased chilling-induced photoinhibition of photosystem II in cotton overexpressing genes encoding chloroplast-targeted antioxidant enzymes. Physiol Plant, 113(3):323-331. DOI:10.1034/j.1399-3054.2001.1130304.x.

Lata C, Prasad M. 2011. Role of DREBs in regulation of abiotic stress responses in plants. $J$ Exp Bot, 62(14):4731-48. DOI:10.1093/jxb/err210.

Lescot M, Déhais P, Thijs G, Marchal K, Moreau Y, Peer Y .V .D, Rouzé, P, Rombauts S. 2002. 
458

459

460

461

462

463

464

465

466

467

468

469

470

471

472

473

474

475

476

477

478

479

480

481

482

483

484

PlantCARE, a database of plant cis-acting regulatory elements and a portal to tools for in silico analysis of promoter sequences. Nucleic Acids Res, 30(1): 325-327. DOI:10.1093/nar/30.1.325.

Li J, Ma W, Zeng P, Wang J, Geng B, Yang J, Cui Q. 2015. LncTar: a tool for predicting the RNA targets of long noncoding RNAs. Brief Bioinform, 16(5):806-12. DOI: 10.1093/bib/bbu048.

Li Q, Lei S, Du K, Li L, Pang X, Wang Z.2016. RNA-seq based transcriptomic analysis uncovers $\alpha$-linolenic acid and jasmonic acid biosynthesis pathways respond to cold acclimation in Camellia japonica. Sci Rep, 6: 36463. DOI:10.1038/srep36463.

Li ZQ, Wang CJ, Yang AG, Ding AM, Feng QF, Xu J, Jiao HP, Shang KY. 2013. Cloning and Subcellular Localization of DXS Gene in Tobacco. J. Anhui Agric. Sci, 41, 11957-11960.

Liu RM, Li FL, Hu GF, Hu BZ. 2011. Cold resistance of transgenic tobacco with CBF3 gene. Journal of Northeast Agricultural University, 1(42):119-23. DOI:10.1631/jzus. A1010009.

Medina J, Bargues M, Terol J, Pérez-Alonso M, Salinas J. 1999. The Arabidopsis CBF gene family is composed of three genes encoding AP2 domain-containing proteins whose expression is regulated by low temperature but not by abscisic acid or dehydration. Plant Physiol, 119(2): 463470. DOI:10.1104/pp.119.2.463.

Mei Z, Wei L, Yu-Ping B, Zi-Zhang W. 2009. Isolation and identification of PNDREB1, a new DREB transcription factor from peanut (Arachis hypogaea L). Acta Agronom Sin, 35(11): 19731980. DOI:10.3724/SP.J.1006.2009.01973.

Moore MJ, Soltis PS, Bell CD, Burleigh JG, Soltis DE. 2010. Phylogenetic analysis of 83 plastid genes further resolves the early diversification of eudicots. Proc Natl Acad Sci USA, 107(10):46234628. DOI:10.1073/pnas.0907801107.

Novillo F, Medina J, Salinas J. 2007. Arabidopsis CBF1 and CBF3 have a different functionthan $\mathrm{CBF} 2$ in cold acclimation and define differentgene classes in the CBF regulon. Proc Natl Acad Sci USA, 104(52): 21002-21007. DOI:10.1073/pnas.0705639105.

Park S, Shi A, Mou B. 2020. Genome-wide identification and expression analysis of the CBF/DREB1 gene family in lettuce. Sci Rep, 10(1): 5733. DOI:10.1038/s41598-020-62458-1.

Potter SC, Luciani A, Eddy SR, Park Y, Lopez R, Finn RD. 2018. HMMER web server: 2018 
485

486

487

488

489

490

491

492

493

494

495

496

497

498

499

500

501

502

503

504

505

506

507

508

509

510

511

update. Nucleic Acids Res, 46(W1):W200-W204. DOI: 10.1093/nar/gky448.

Stockinger EJ, Gilmour SJ, Thomashow MF. 1997. Arabidopsis thaliana CBF1 encodes an AP2 domain-containing transcriptional activator that binds to the C-repeat/DRE, a cis-acting DNA regulatory element that stimulates transcription in response to low temperature and water deficit. Proc Natl Acad Sci USA, 94(3):1035-1040. DOI:10.1073/pnas.94.3.1035.

Tamura K, Stecher G, Peterson D, Filipski A, Kumar S. 2013. MEGA6: Molecular evolutionary genetics analysis version 6.0. Mol Biol Evol, 30(12): 2725-2729. DOI:10.1093/molbev/mst197.

Tang M, Liu X, Deng H, Shen S. 2011. Over-expression of JcDREB, a putative AP2/EREBP domain-containing transcription factor gene in woody biodiesel plant Jatropha curcas, enhances salt and freezing tolerance in transgenic Arabidopsis thaliana. Plant Sci, 181(6): 623-31. DOI:10.1016/j.plantsci.2011.06.014.

Tayeh N, Bahrman N, Sellier H, Bluteau A, Blassiau C, Fourment J, Bellec A, Debellé F, LejeuneHénaut I, Delbreil B. 2013. A tandem array of CBF/DREB1 genes is located in a major freezing tolerance QTL region on Medicago truncatula chromosome 6. BMC Genomics, 14(1):814. DOI:10.1186/1471-2164-14-814.

Tillett RL, Wheatley MD, Tattersall EA, Schlauch KA, Cramer GR, Cushman JC. 2012. The Vitis vinifera C-repeat binding protein 4 (VvCBF4) transcriptional factor enhances freezing tolerance in wine grape. Plant Biotechnol J, 10(1):105-124. DOI: 10.1111/j.14677652.2011.00648.x.

Wang PJ, Chen XJ, Guo YC, Zheng YC, Yue C, Yang JF, Ye NX. 2019. Identification of CBF Transcription Factors in Tea Plants and a Survey of Potential CBF Target Genes under Low Temperature. Int J Mol Sci , 20(20): 5137. DOI:10.3390/ijms20205137.

Würschum T, Longin CFH, Hahn V, Tucker MR, Leiser WL. 2017. Copy number variations of CBF genes at the Fr-A2 locus are essential components of winter hardiness in wheat. Plant $J$, 89(4):764-773. DOI:10.1111/tpj.13424.

Xie C, Mao X, Huang J, Ding Y, Wu J, Dong S, Kong L, Gao G, Li CY, Wei L. 2010. KOBAS 2.0: a web server for annotation and identification of enriched pathways and diseases. Nucleic 
512 Acids Research, 39: W316-W322. DOI:10.1093/nar/gkr483.

513 Zhao C, Zhang Z, Xie S, Si T, Li Y, Zhu JK. 2016. Mutational Evidence for the Critical Role of

514 CBF Transcription Factors in Cold Acclimation in Arabidopsis. Plant Physiology, 171(4): 2744515 2759. DOI:10.1104/pp.16.00533. 


\section{Table $\mathbf{1}$ (on next page)}

Table 1 . The physicochemical properties for the 4 CBF gene family members in $C$. japonica. 


\begin{tabular}{|c|c|c|c|c|c|c|c|c|}
\hline Gene name & Full length(kb) & CDS(bp) & $\begin{array}{c}\text { Number of } \\
\text { amino acids }\end{array}$ & $\begin{array}{c}\text { Molecular Weight } \\
\text { (kDa) }\end{array}$ & Theoretical pl & $\begin{array}{c}\text { Grand average of } \\
\text { hydropathicity }\end{array}$ & $\begin{array}{c}\text { Aliphatic } \\
\text { index }\end{array}$ & $\begin{array}{c}\text { Instability } \\
\text { index }\end{array}$ \\
\hline$C j C B F 1$ & 1.82 & 837 & 278 & 30282.91 & 4.92 & -0.533 & 66.08 & 53.31 \\
\hline$C j C B F 2$ & 1.25 & 540 & 179 & 19449.25 & 9.18 & -0.411 & 74.19 & 51.14 \\
\hline$C j C B F 3$ & 1. 42 & 741 & 246 & 26655.03 & 5.25 & -0.407 & 66.71 & 46.86 \\
\hline CjCBF4 & 1.35 & 720 & 239 & 26526.23 & 5.50 & -0.328 & 74.35 & 48.40 \\
\hline
\end{tabular}


Figure 1

Fig. 1. Multiple sequence alignment of CBFs gene and Conservative motif analysis

(A) The AP2 domain is represented in the yellow box. (B) The architecture of the 10 conserved protein motifs predicted via MEME analysis. Each motif is represented in a different color (Motif 1-10).

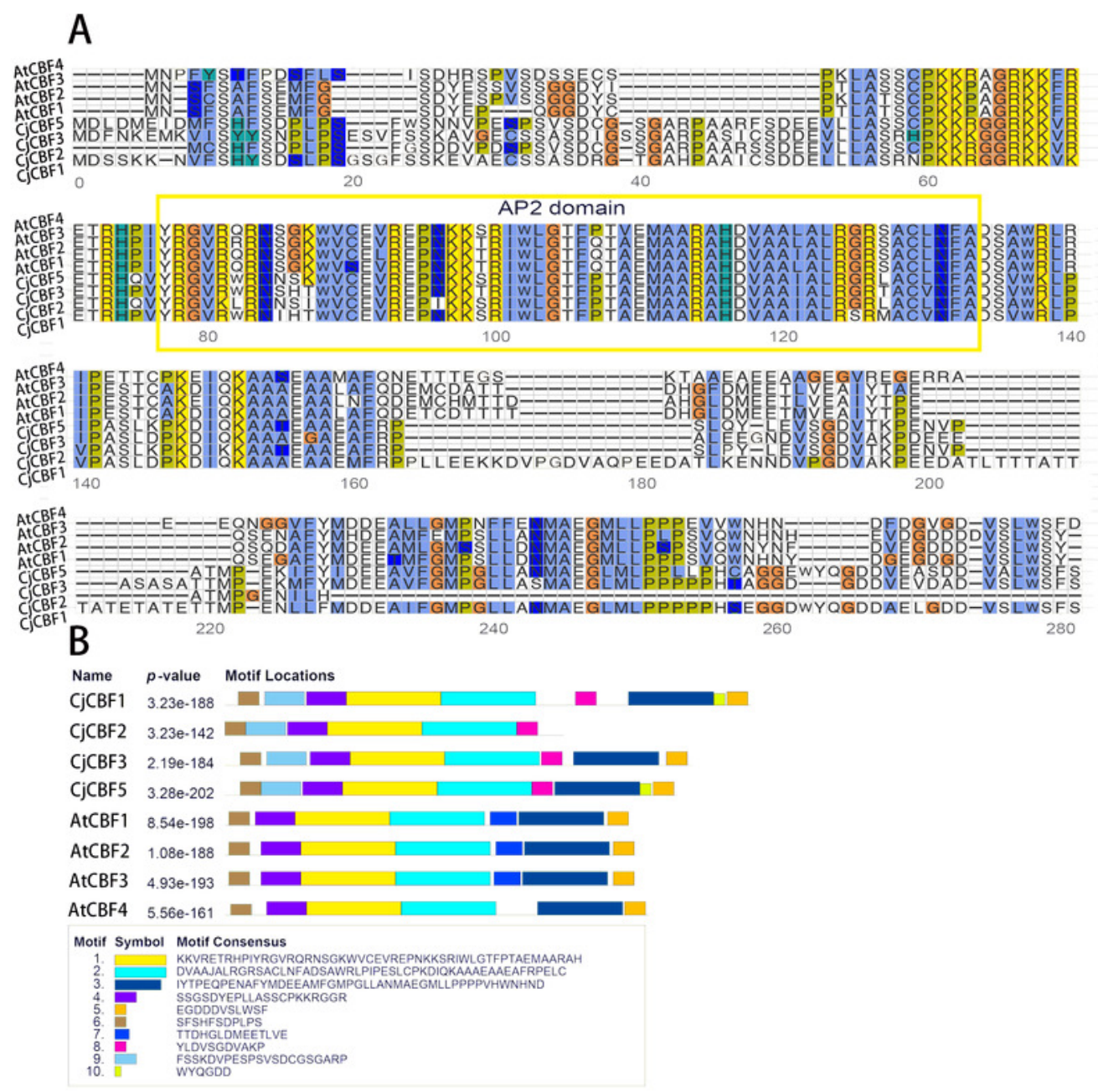




\section{Figure 2}

Fig. 2. Phylogenetic analysis of CBFs

Maximum Likelihood phylogenetic trees of the protein sequences of 424 genes encoding CBFs from 19 angiosperms. camellia (Cj: Camellia japonica) are marked red in the evolutionary tree, and Arabidopsis (At: Arabidopsis thaliana) are marked blue in the evolutionary tree. The other species are: Eucalyptus (Eug: Eucalyptus grandis); soybean (Gm: Glycine max); Cucumber (Cs: Cucumis sativus); Chili Peppers (Ca: Capsicum annuum); Cotton (Gh: Gossypium hirsutum); potato (St: Solanum tuberosum); western balsam poplar (Pt: Populus trichocarpa); Kiwi fruit (Ac: Actinidia Chinensis); Morning glory (Pn: Pharbitis nil); Sunflower (Ha: Helianthus annuus); Rubber Tree (Hb: Hevea brasiliensis); sesame (Si: Sesamum indicum); Carrot (Dc: Daucus carotavar.sativa); Medicago (Mt: Medicago truncatula); Brachypodium distachyon (Bd: Brachypodium distachyon); rice (Os: Oryza sativa); oil palm (Eg: Elaeis guineensis). 


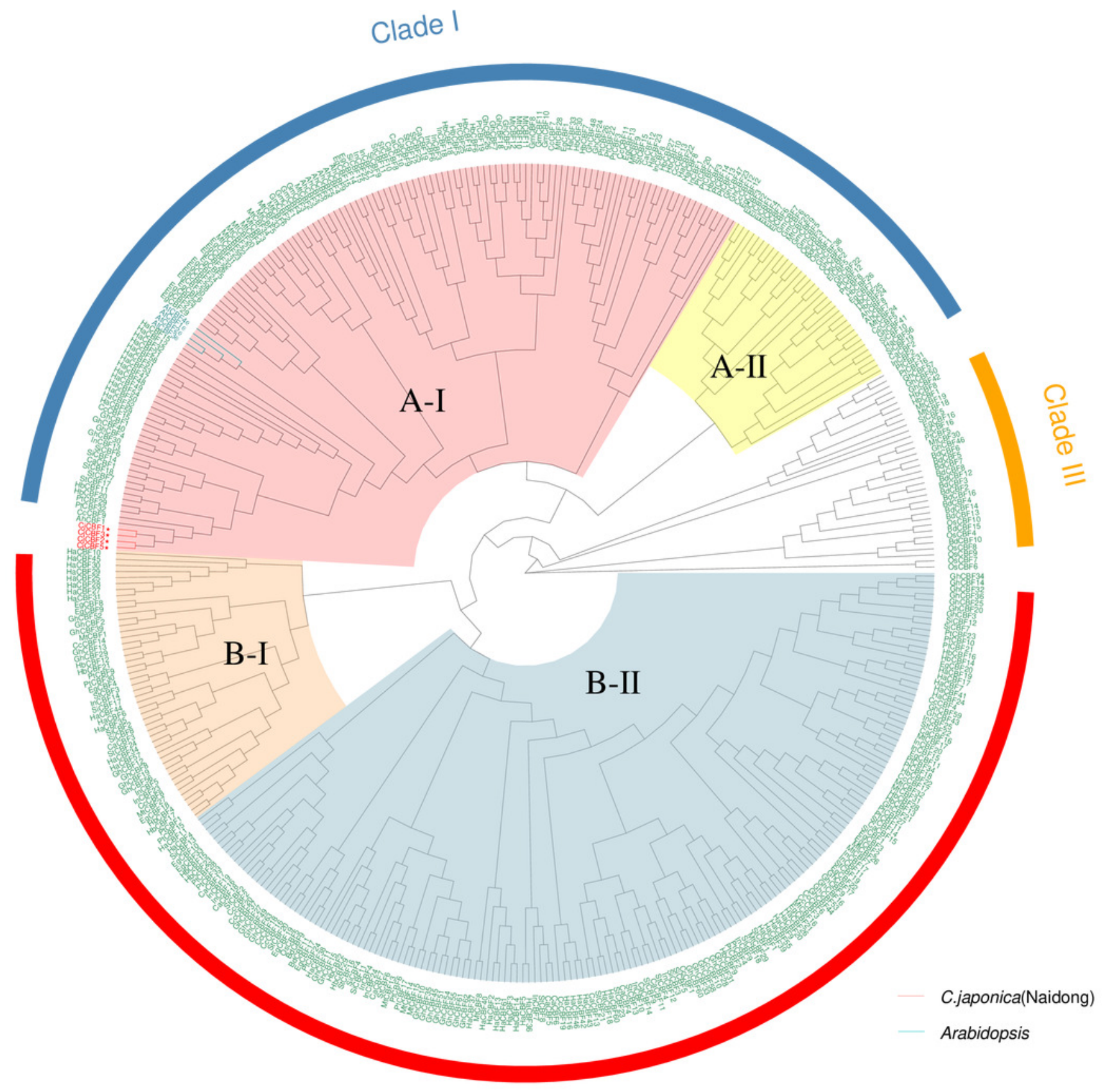

Clade II 
Figure 3

Fig. 3. $\mathrm{GO}$ analysis

The GO enrichment results of CjCBFs based on KOBAs. The enrichment degree increases with the $-\log 10$ (pvalue) value.

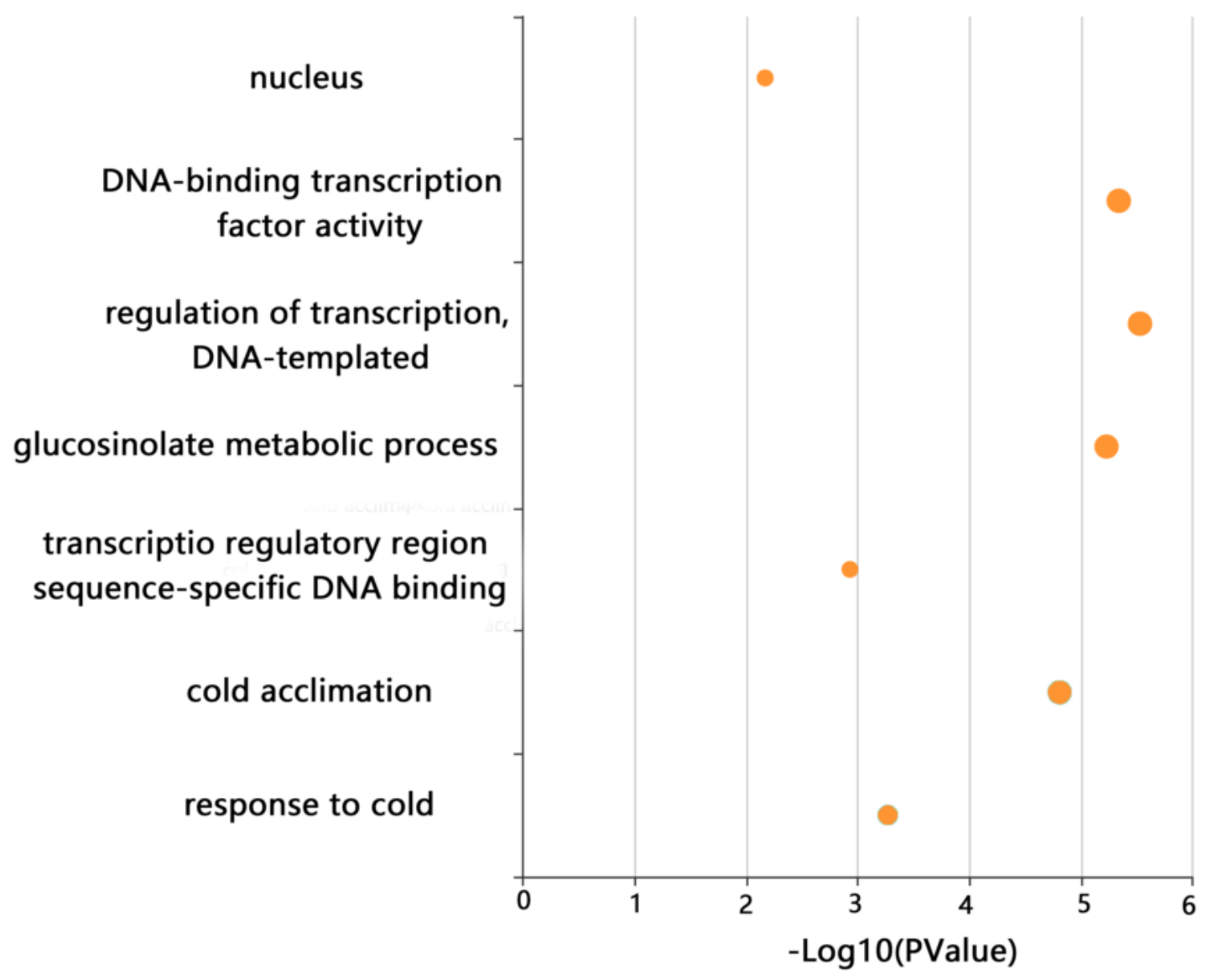




\section{Figure 4}

Fig. 4. Expression analysis based on the transcript group data

(A) Expression pattern of CjCBFs in response to cold Oh(CK), 12h(T1), 24h(T2), 72h(T3)

treatments, The RNA-seq data were normalized based on the mean expression value ( $\log 2$ fold change, $\log 2 \mathrm{FC}$ ) of each gene, green and blue boxes indicate high and low expression levels, respectively. (B) Correlation analysis of four CjCBFs with different low temperature treatment groups (cyan region connects CjCBFI and four treatment groups, yellow region connects CjCBF5 and four treatment groups, brown region connects CjCBF3 and four treatment groups, purple region connects CjCBF2 and four treatment groups), The wider the region, the higher the correlation. (C) Verification of the CjCBFs expression in the transcriptome via qRT-PCR. (D) Differential expression heat map of the IncRNAs that regulate CjCBFs based on the full-length transcriptome data. The data were normalized based on the mean expression value (log2fold change, $\log 2 \mathrm{FC}$ ) of each IncRNAs, green and blue boxes indicate high and low expression levels, respectively. Yellow, green and blue groups represent the regulation of $C j C B F 2, C j C B F 1$, and CjCBF5, respectively. 
A

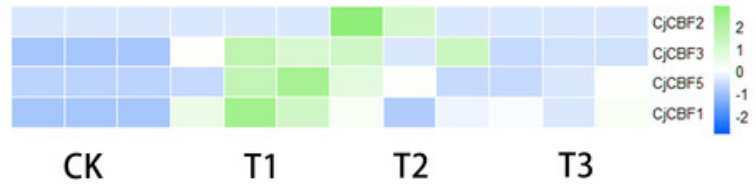

C
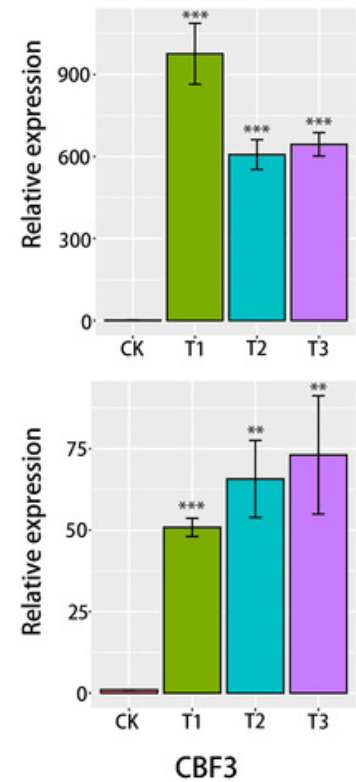
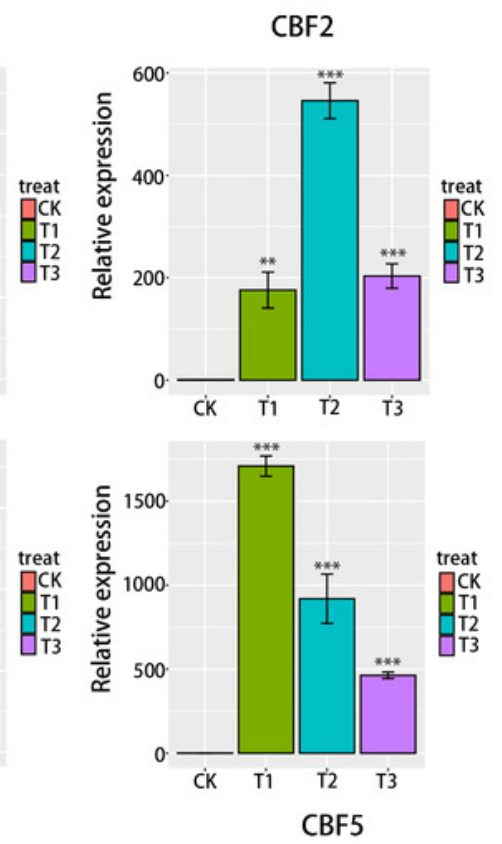
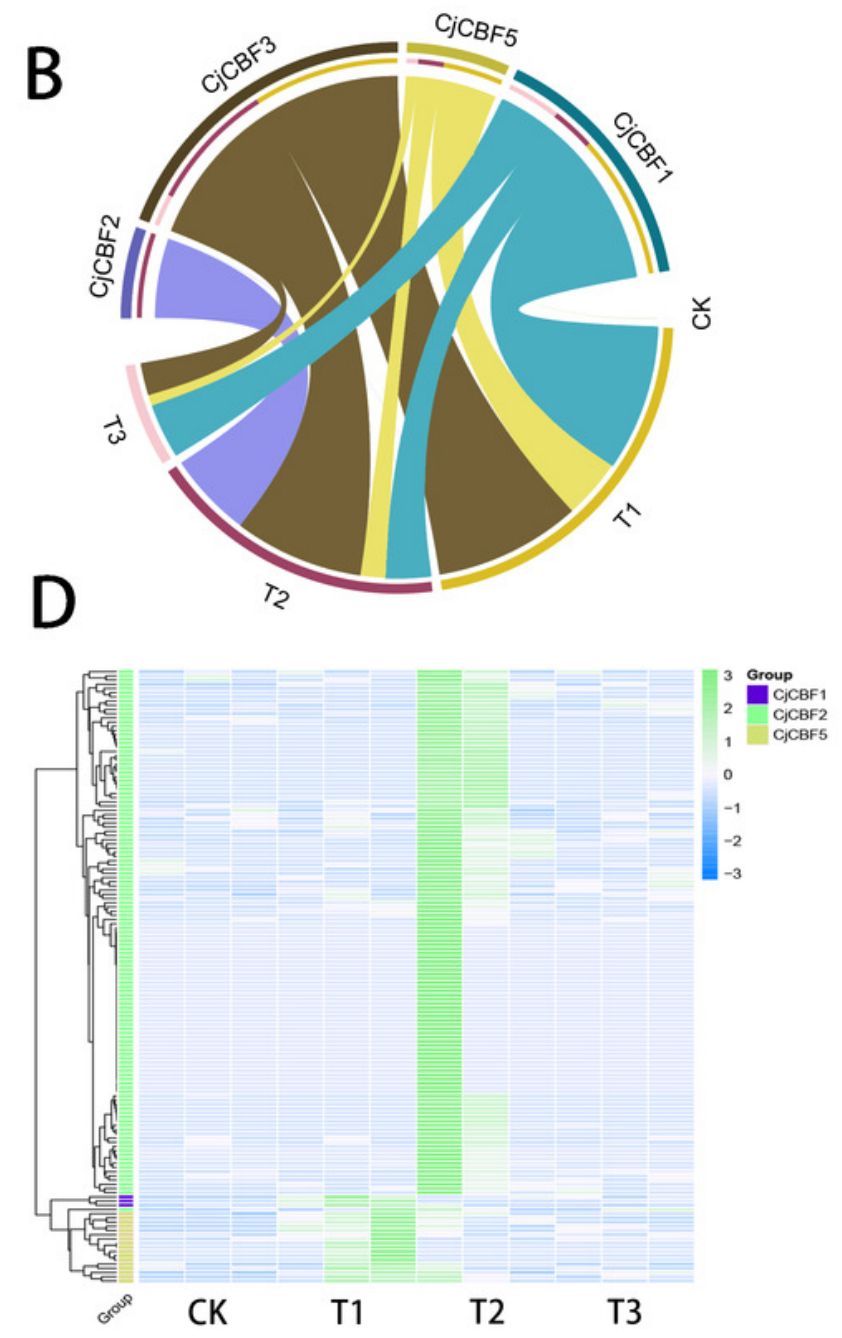


\section{Figure 5}

Fig. 5. Protein interaction identification and subcellular localization

(A)Self-activation verification. White colony indicates the ability of yeast to grow on the deficient medium, blue color indicates that the self-activating activity of CjCBFs was not able to grow on the deficient medium supplemented with AbA. This points towards the inhibition of the self-activating activity. (B)Yeast two-hybrid. Yeast cells exhibit normal growth on the nutrient-deficient medium, indicating the interaction between CjCBF1, CjCBF2 and CjCBF5. (C)Subcellular localization of Super1300, CjCBF1, CjCBF2 and CjCBF5 in Nicotiana benthamiana. In bright, the black line is the outline of the cell. For eGFP, the green region is the expression site of the plasmid with GFP fluorescent protein and the CjCBFs gene. 
A
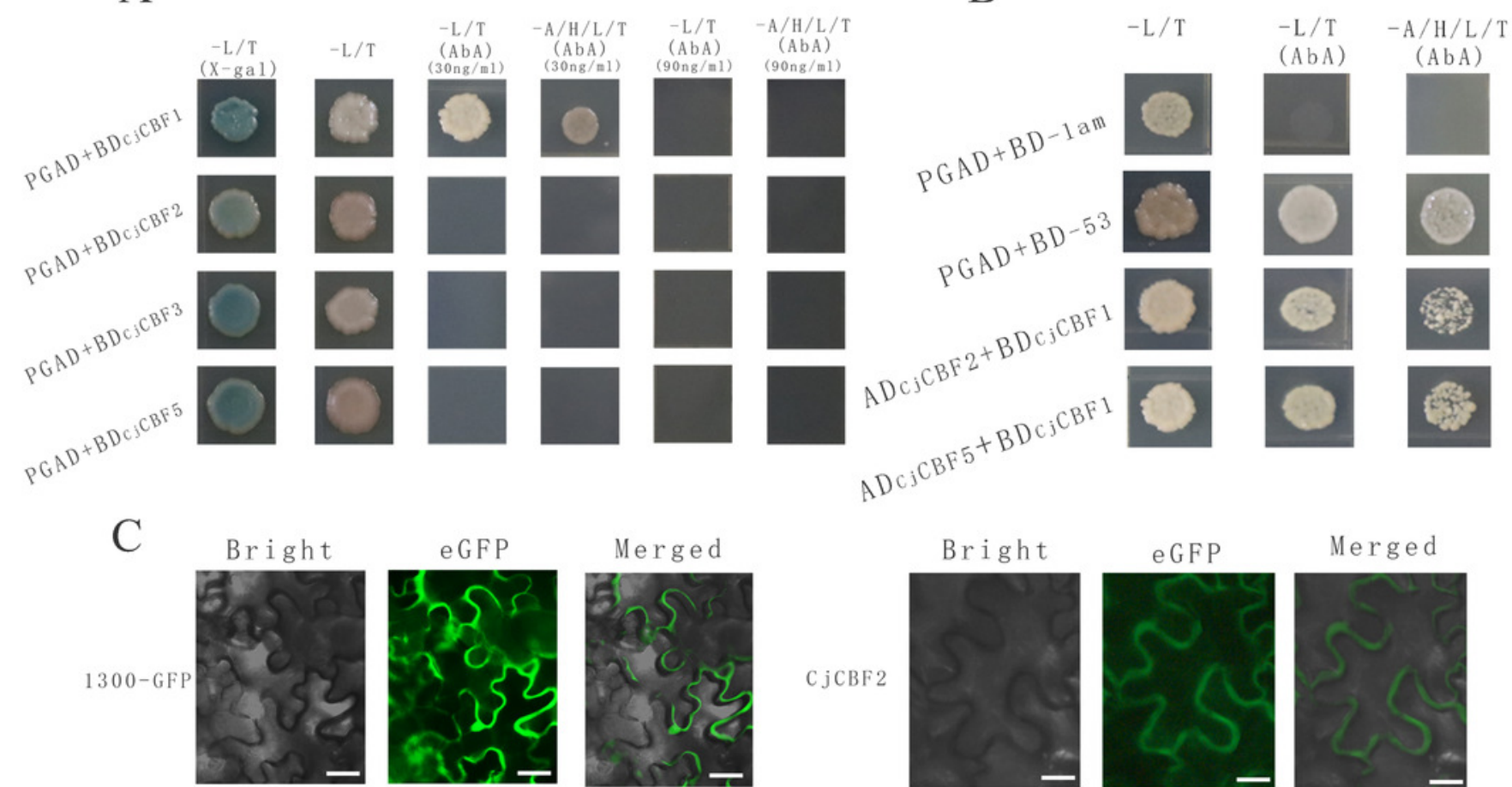

Merged

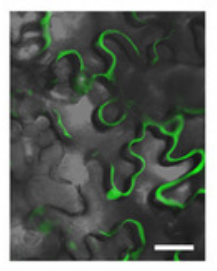

Bright

eGFP

Merged

C jCBF 2
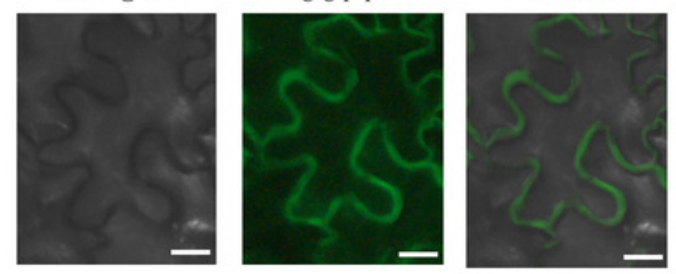

C jCBF 1
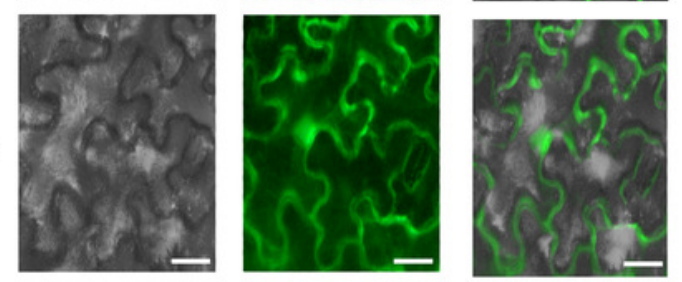

C jCBF 5
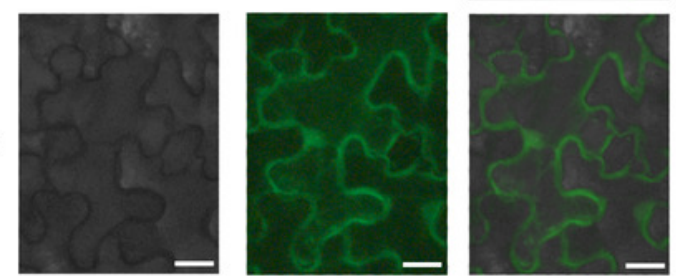


\section{Figure 6}

Fig. 6. Organ-specific expression of four CjCBFs

(A)-(E) We measured the relative expression amounts of 4 CjCBFs in each tissue type for A, B, $C, D, E$, and set the expression amounts of CjCBF1 in the CK group to 1 for each tissue. This was then used to calculate the relative expression amounts of other CjCBFs for all tissue types. (F)-(I) We measured the relative expression of each CjCBFs in 5 tissues got $F, G, H, I$, and set the expression of each CjCBFs in the root of the CK group to 1 . This was then used to calculate the relative expression of each CjCBFs in other tissues.
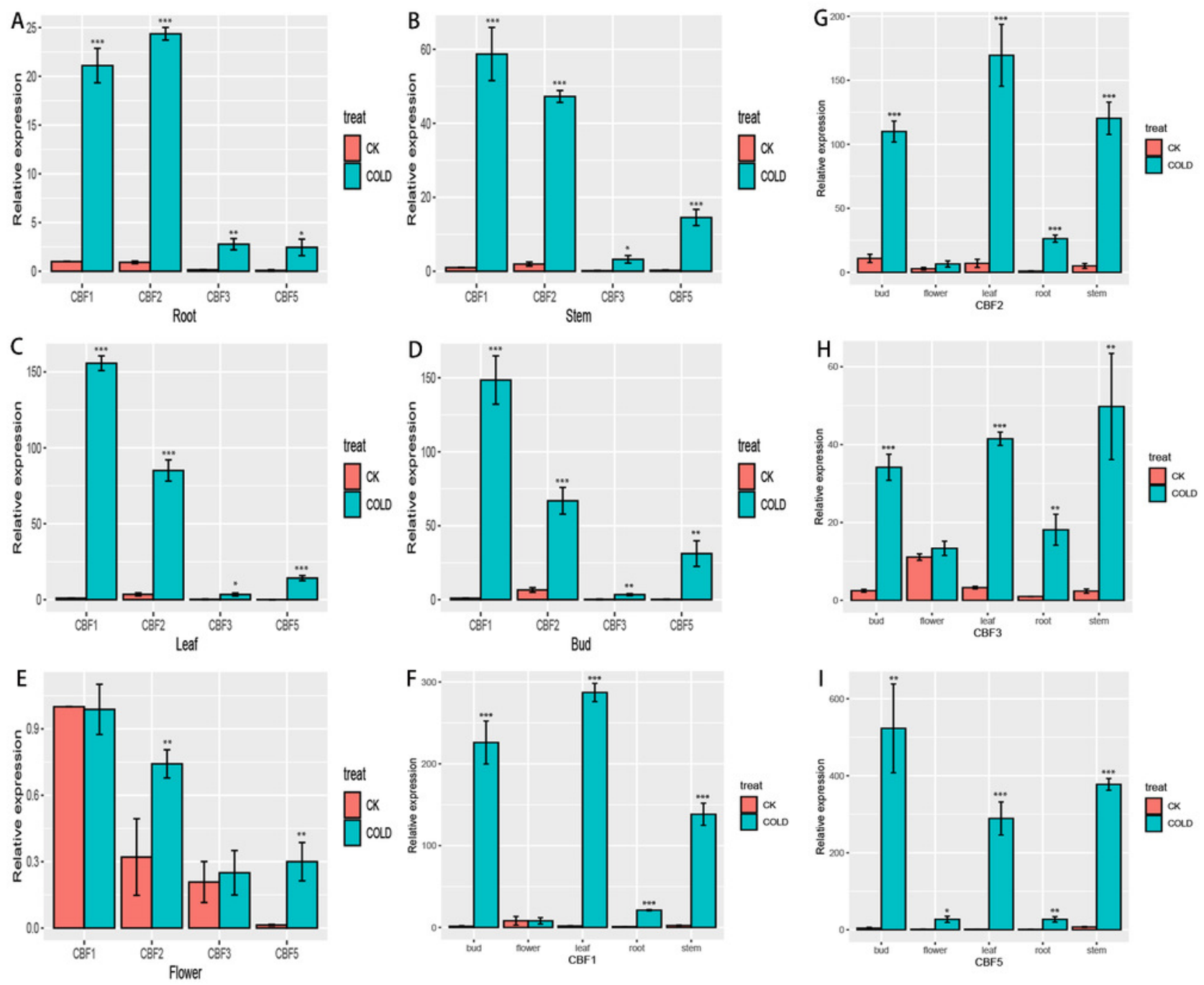
Figure 7

Fig. 7. Prediction of cis-elements in the CjCBF1 promoters.

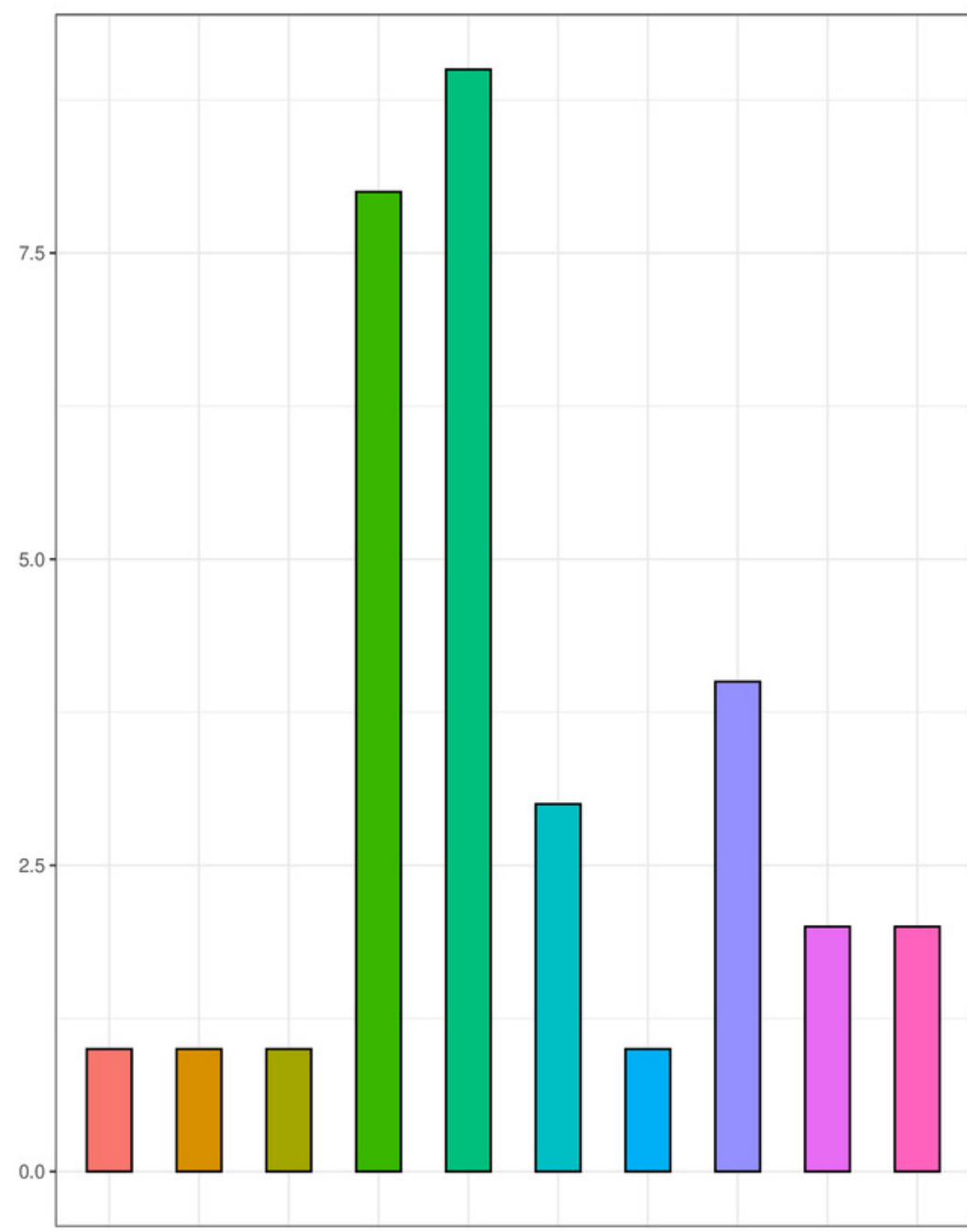

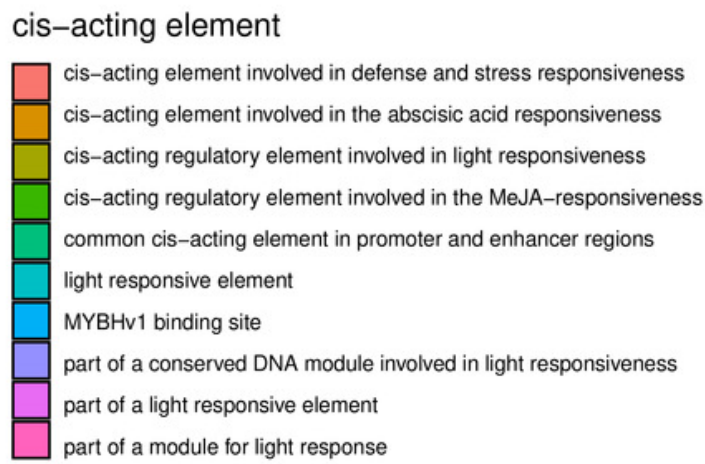


Figure 8

Fig. 8. Response of CBFs to multiple stresses

(A)Relative expression of CjCBFs under the heat and hot (no-light) treatments. The expression of CjCBFs in each CK group was set to 1. (B)Relative expression of CjCBFs under the cold and cold (no-light) treatments. The expression of CjCBFs in each CK group was set to 1. (C) Relative expression of CjCBFs in the drought treatment. The expression of CjCBFs in each CK group was set to 1 .

A

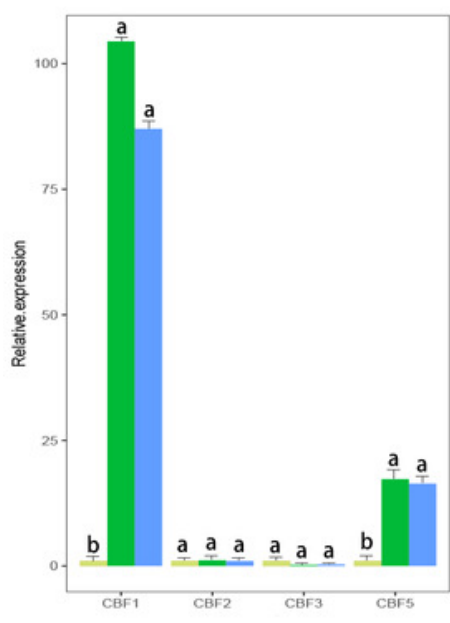

B

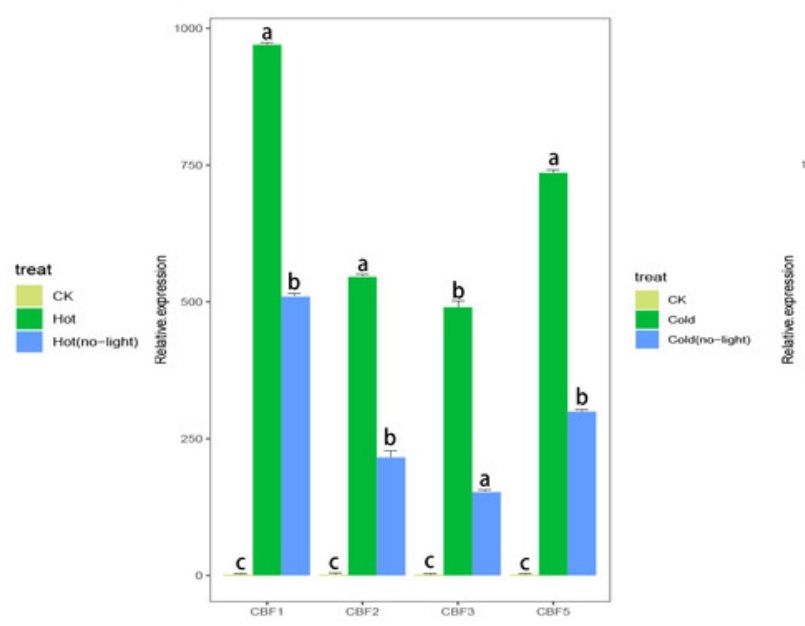

C

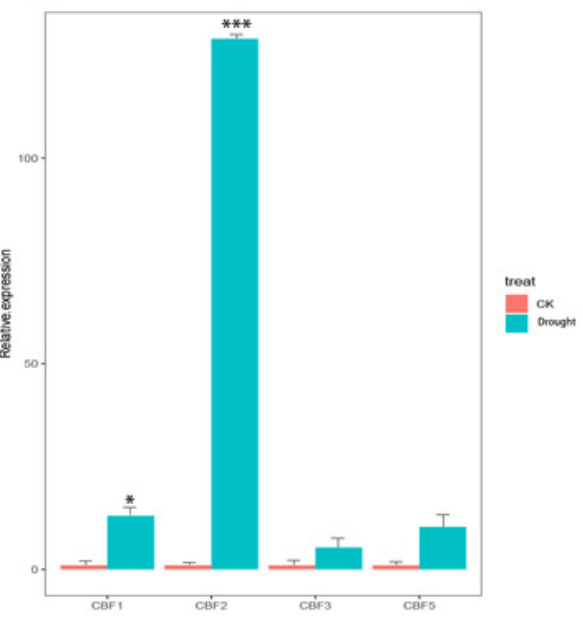

